\title{
La production des innovations sociales : une analyse par le modèle de l'écologie créative
}

\author{
The production of social innovations in the light of the model of creative \\ ecology
}

\author{
Paul Muller ${ }^{1}$ \\ ${ }^{1}$ Université de Lorraine, Université de Strasbourg, CNRS, BETA, 54000, Nancy, France, paul.muller@univ-lorraine.fr
}

\begin{abstract}
RÉSUMÉ. La notion d'innovation sociale a fait l'objet d'un intérêt croissant au cours des dernières années. En s'attachant surtout à la définir et d'en étudier les effets, le problème des processus créatifs collectifs associés à sa production a été peu étudié. Nous mobilisons le modèle de l'écologie créative afin de répondre à ce vide théorique. Ce modèle, mettant en avant différents niveaux économiques (upper-, middle-, underground), nous permet de mettre en avant le fait que les innovations sociales peuvent être produites suivant deux logiques complémentaires. Suivant une logique top-down, les acteurs à leur initiative sont localisés dans l'upperground. Les innovations ainsi créées ont une capacité de diffusion élevée, mais peuvent avoir un apport limité car elles sont soumises à un arbitrage entre viabilité économique et impact social. A l'inverse, les innovations sociales produites suivant une logique bottom-up émanent d'acteurs de l'underground. Coproduites avec leurs bénéficiaires, elles sont à même de répondre à leurs besoins spécifiques mais éprouvent des difficultés à se diffuser à plus grande échelle. Du fait de leurs limites respectives, les logiques topdown et bottom-up sont fréquemment mises en œuvre de manière concourante au sein des écologies créatives. Ceci peut soulever des problèmes de gouvernance dont la résolution fait intervenir les structures de l'économie sociale et solidaire, au travers de leur fonction d'intermédiation d'innovation.

ABSTRACT. The concept of social innovation has gained significant traction over the past few years. Academic work is mostly focused on defining and delineating the concept and on grasping its socio-economic importance. The issue of the collective creative processes associated with its production has therefore been overlooked. We will address this theoretical gap through recourse to the model of creative ecology. By highlighting the coexistence of different economic strata, (upper-, middle-, underground), this model allows us to argue that social innovation may be produced through two complementary logics. Social innovations produced through a top-down logic originate in the upperground. They benefit from a high diffusion potential. However, their social outcome may be limited, due to the fact that they are subject to a trade-off between social impact and economic viability. On the other hand, social innovations produced through a bottom-up logic stem from the underground. As they are coproduced with beneficiaries, they are most able to address their specific social needs, but may be difficult to scale up. This is why both logics can be at work simultaneously, which, in turn, raises governance issues. We will argue that social economy organizations contribute to solving these issues, as they act as innovation intermediaries.
\end{abstract}

MOTS-CLÉS. Innovation sociale, écologie créative, économie sociale et solidaire, intermédiation.

KEYWORDS. Social innovation, creative ecology, social economy, intermediation.

\section{Introduction}

Bien qu'apparue dès la fin du XIXe siècle [GOD 12], la notion d'innovation sociale n'a fait l'objet d'un intérêt significatif et grandissant dans la littérature en économie et gestion que depuis la seconde moitié des années 2000 (figure 1). Jusqu'alors, il a été essentiellement traité des innovations à dimension économique : innovations technologiques, de services, organisationnelles, sur les modèles économiques. De manière générale, leur objectif est de permettre aux firmes et aux territoires d'améliorer leur compétitivité ou de créer de nouvelles opportunités de marché. Cependant, une focalisation exclusive sur ces dernières donne lieu à deux types de limites. Premièrement, elle restreint l'analyse aux innovations évoluant au sein de et ayant un impact sur les systèmes régis par un ordre marchand ou par l'action publique. De ce fait, sont ignorées celles qui produisent essentiellement leurs effets via d'autres mécanismes de coordination économique tels que la réciprocité [LAV 01]. Deuxièmement, les processus de destruction créatrice, indissociables des dynamiques d'innovation, notamment technologiques, ont été essentiellement mis en avant dans leur dimension économique (compétences professionnelles, emploi...) tout en minorant leur dimension sociale : remise en cause 
des modes d'organisation sociale et de socialisation, du statut social des personnes, etc. La notion d'innovation sociale permet précisément de tenir compte de ces limites analytiques et vise à mieux comprendre comment compenser les possibles conséquences sociales négatives des processus de destruction créatrice des innovations à dimension économique.

Si nous nous intéressons plus particulièrement au problème de la production des innovations sociales, nous constatons que la recherche existante a essentiellement traité des facteurs associés à leur mise en œuvre, ainsi que les caractéristiques des personnes à leur origine. Elle a peu abordé le problème des processus créatifs collectifs impliqués dans leur production et leur diffusion, alors que ce thème est considéré comme central dans la littérature sur les innovations à dimension économique [voir BAT 17] [BUR 13]. L'objectif de cet article est, précisément, de mobiliser les apports de la littérature sur la créativité pour parvenir à mieux comprendre les processus collectifs associés à la production et la diffusion des innovations sociales. Pour ce faire, nous mobilisons en particulier le modèle de l'écologie créative, développé par Patrick Cohendet et Laurent Simon [SIM 09] [COH 10] [GRA 13]. Inspiré des travaux de Florida [FLO 05] [STO 06], ce cadre d'analyse est pertinent car il met au centre de l'analyse les dynamiques collectives de parties prenantes diverses, caractérisées par différentes positions, propriétés socio-économiques et rationalités. Un intérêt majeur de ce modèle pour notre recherche est qu'il permet la mise en avant de différentes modalités de production et de gouvernance des innovations sociales. Un enjeu important des écologies créatives en lien avec les innovations sociales revient dès lors à les faire cohabiter. De ce point de vue, nous verrons que les structures de l'économie sociale et solidaire (ESS) jouent un rôle d'intermédiation essentiel.

Nous proposerons, dans une deuxième partie, une définition synthétisant les principales recherches dans le domaine. Dans une troisième partie, nous présenterons le modèle de l'écologie créative. Dans une quatrième partie, nous aborderons la production des innovations sociales au sein des écologies créatives, en insistant sur les différentes logiques de gouvernance des processus créatifs associés. Nous développerons dans une cinquième partie les rôles joués par les structures de l'économie sociale et solidaire (ESS) dans ces derniers. En conclusion, après un rappel de notre argumentation, nous soulignerons l'enjeu majeur de la gouvernance et de la professionnalisation des structures de l'ESS sur sa capacité à développer et à porter les innovations sociales.

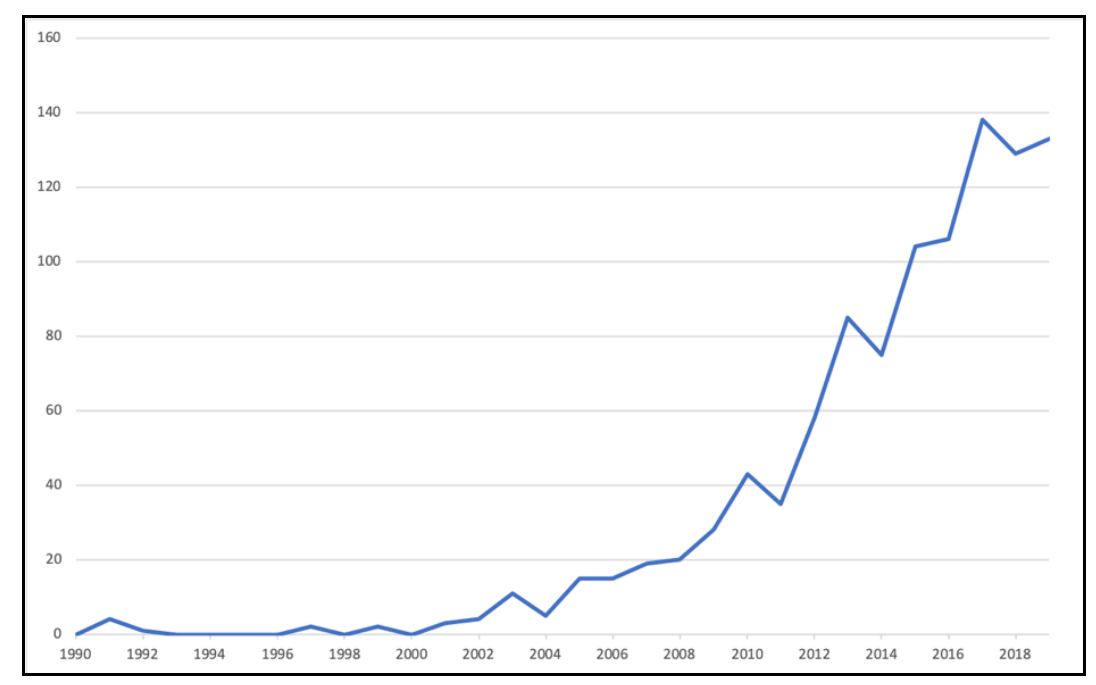

Figure 1. Nombre annuel de publications comptant les termes « social innovation » ou "innovation sociale " dans le titre et/ou le résumé, recensées dans la base de données Business Source Premier pour la période 1990-2019.

\section{Définir l'innovation sociale}

La notion d'innovation sociale a été définie de différentes manières, ce qui reflète la multiplicité des champs de recherche qui l'ont investie. Il peut être notamment mis en avant les littératures sur les 
grands défis sociaux et technologiques, en psychologie communautaire, sur la créativité ou sur le développement territorial.

La littérature traitant des innovations sociales pour résoudre les grands défis sociotechnologiques (les grands challenges) la définit « comme des ruptures dans les manières dont les acteurs envisagent de nouvelles pratiques possibles » [CHO 15][YOU 19]. Faisant suite à une démarche intentionnelle impliquant une relation réciproque entre les acteurs et les structures [CAJ 14], leur objectif est d'induire des changements sociaux, le cas échéant en interaction avec d'autres formes d'innovation, dans une perspective de développement durable [VAN 16].

Pour leur part, les recherches en psychologie communautaires mettent en avant le fait que les innovations sociales ont pour but d'introduire des changements sociaux au niveau des communautés et d'améliorer la qualité de vie de leurs membres par le biais des innovations sociales [HAZ 03]. Les innovations sociales sont dès lors associées aux processus menant aux changements plutôt qu'aux résultats, ce qui est traité dans la littérature sur l'innovation dans les enjeux sociotechnologiques.

Dans la littérature sur la créativité, l'innovation sociale se réfère à la génération et la mise en œuvre d'idées nouvelles concernant l'organisation des activités interpersonnelles ou des interactions sociales [MUM 03]. Elle se traduit, soit par la création d'institutions sociales, ou d'idées inédites concernant la gouvernance ou le développement de mouvements sociaux, soit par la création de nouveaux processus et procédures en vue de structurer le travail collaboratif, ainsi que l'introduction de pratiques sociales nouvelles au sein d'un groupe [MUM 02]. Cette littérature a avant tout étudié les facteurs et caractéristiques personnelles et contextuelles qui sont à l'origine des innovations sociales, délaissant les processus créatifs à l'œuvre dans leur production.

Enfin, la littérature liant innovation sociale et développement territorial la définit comme «le développement de réponses nouvelles à des besoins exprimés par des groupes sociaux particuliers, et qui ne peuvent pas être satisfaits, voire reconnus » [AND 09 p.149] par le marché et par les institutions publiques. Nous adopterons cette définition dans la suite de cet article. Son intérêt réside dans le fait qu'elle opère une synthèse de trois parmi les caractéristiques principales des innovations sociales, les distinguant des innovations à dimension économique [BOU 06][HAR 06][HIL 04][MOU 09][POL 09]. Premièrement, l'objectif de l'innovation sociale réside avant tout dans l'amélioration des conditions de vie des groupes sociaux concernés par la fourniture de solutions adaptées à leurs besoins et problèmes. Ceci implique notamment qu'elle ne peut pas se fonder uniquement sur un produit ou service isolé, mais sur une solution globale, pouvant impliquer un ensemble de produits et/ou services adaptés.

Deuxièmement, sa finalité principale n'étant pas d'ordre économique, les acteurs économiques traditionnels (firmes, État et collectivités) ne sont pas nécessairement en mesure de répondre de manière directe au besoin exprimé, soit parce qu'il n'y a pas de perspectives de profits suffisants à réaliser, soit parce que la nature du besoin est telle qu'il est impossible de proposer une réponse adaptée. Ceci ne préjuge pas pour autant d'une absence totale d'acteurs ni de mécanismes de coordination marchande ou redistributive, qui peuvent intervenir, par exemple au travers d'actions de financement des initiateurs de projets d'innovation sociale. En outre, dans un contexte de raréfaction des financements publics, ces derniers peuvent être incités à mener des activités commerciales (ventes de biens et services...) en vue d'assurer le financement de l'innovation sociale, voire de chercher à valoriser économiquement l'innovation sociale. Cette relation complexe entre innovation sociale et valorisation économique, faite de tensions et de complémentarités, peut être notamment montrée dans le cas du commerce équitable [LEV 08].

Troisièmement, l'innovation sociale est, par nature, issue de processus collectifs requérant la collaboration avec les publics concernés. Elle est, par conséquent, encastrée dans un contexte social donné qui est souvent local. En outre, elle conduit souvent à une remise en cause des règles socioéconomiques, voire des modèles de pensée collectifs en vigueur dans les milieux concernés. Ceci est 
notamment visible dans le cas des monnaies locales, qui participent à des questionnements plus larges sur les statuts de la monnaie et du travail prévalant au sein des sociétés capitalistes occidentales.

Enfin, un trait distinctif de l'innovation sociale par rapport aux innovations à dimension économique est qu'elle n'est pas nécessairement associée à un critère de nouveauté absolue mais peut consister en la mobilisation et l'adaptation de pratiques anciennes au contexte social actuel. Néanmoins, elle peut s'appuyer sur ces autres formes d'innovation, en particulier technologiques et de service. Il peut notamment être mentionné les innovations frugales qui sont, bien souvent, des innovations technologiques ou de services partant de l'expression d'un besoin social [ZES 11].

Il peut être cependant souligné qu'une faiblesse récurrente de la littérature sur les innovations sociales se situe dans le fait que les processus associés à leur production sont encore assez mal compris. La littérature sur la créativité, en particulier, a surtout mis en exergue des caractéristiques individuelles ou, éventuellement, contextuelles pour l'expliquer. Bien que considérée comme centrale dans leur mise en œuvre, notamment dans les littératures en psychologie communautaire et en développement territorial, la dimension collective dans leur production n'a été, à notre sens, que peu abordée. De plus, il n'y est pas considéré les relations complexes pouvant se développer entre innovation sociale et innovation à dimension économique. Ceci sera au cœur de notre discussion appliquant le modèle de l'écologie créative dans la production des innovations sociales.

\section{Présentation du modèle de l'écologie créative}

Notre recherche s'appuie sur le modèle de l'écologie créative développé par Patrick Cohendet, David Grandadam et Laurent Simon [COH 10] [COH 14] [GRA 13]. Il met en avant le fait que les processus créatifs, précurseurs des innovations, se produisant au sein d'un écosystème (territoire, industrie...), ne se résument pas aux activités d'innovation menées au sein des firmes. Faisant suite aux travaux de Florida [FLO 05][STO 06], le modèle considère les processus créatifs reposant sur des interactions entre des acteurs relevant de différentes strates économiques [COH 10] : l'upperground, le middleground et l'underground.

L'upperground concerne l'ensemble des acteurs publics et privés considérés comme institutionnels [SIM 09]. Il s'agit du niveau privilégié pour la valorisation économique des innovations, qu'elles soient technologiques, immatérielles ou organisationnelles, au travers des mécanismes marchands ou redistributifs. L'upperground compte les institutions traditionnelles, qu'elles soient publiques (administrations, collectivités, organismes de formation et de recherche...) ou privées comme les entreprises de droit commercial.

L'underground regroupe des acteurs individuels créatifs : artistes amateurs et personnes créatives [FIS 11]. Ils contribuent au bouillonnement créatif à l'origine de nouvelles idées. Ce niveau se caractérise par des activités créatives, artistiques et culturelles se déroulant en dehors de toute organisation liée à une activité marchande ou redistributive, en ayant lieu «dans les garages». L'underground inclut, par exemple, des artistes, militants et activistes pratiquant une activité créative à titre privé et confidentiel. À l'inverse de l'upperground, les mécanismes marchands et redistributifs sont absents de l'underground, au bénéfice de mécanismes économiques basés sur la réciprocité. Les activités menées dans l'underground s'affranchissent donc des contraintes de valorisation économiques et bénéficient d'une plus grande latitude pour l'expérimentation. Les acteurs de ce niveau sont souvent amenés à développer des utopies ou de nouvelles visions de la société. La contrepartie de cette autonomie créative réside, pour ces acteurs, dans la difficulté de valoriser économiquement les idées créatives et de les diffuser à une large échelle.

Le middleground agrège les idées créatives provenant de l'underground. Il forme un «sas » de circulation des idées créatives développées dans le cadre des activités d'exploration de l'underground en vue de leur valorisation par les mécanismes marchands et redistributifs au sein de l'upperground 
[SAR 17]. Le middleground se caractérise, à l'instar de l'upperground, par la dimension collective des activités cognitives menées [MEH 17]. La diffusion des idées créatives se produit à l'occasion des interactions entre les agents économiques. À ce titre, quatre mécanismes du middleground entrent en jeu : les lieux et les plateformes, ainsi que les événements et projets.

- Les lieux forment des plateformes physiques constituant le cadre des interactions [LES 17]. Il s'agit de dispositifs de proximité géographique permanente comme des espaces de coworking, fablabs ou bars et cafés, ou temporaires, comme des conventions, festivals ou salons [TOR 08]. Ils peuvent aussi être virtuels, à l'instar des forums internet [BOU 09].

- À l'instar des lieux, les espaces forment des espaces cognitifs formant le cadre des interactions. Nous pouvons y trouver des communautés, qu'elles soient épistémiques ou de pratique, ainsi que des structures, souvent de petite taille, sous statut commercial ou associatif, qui se démarquant des structures de l'upperground par le fait que leur objectif principal ne réside pas dans la recherche de profits. En particulier, ainsi que nous le verrons ultérieurement, les structures de l'économie sociale et solidaire (ESS) y jouent un rôle essentiel.

Les lieux et espaces, en tant que plateformes de circulation des idées et connaissances, doivent, à leur tour, être activés par deux autres mécanismes du middleground: les événements et les projets [LAN 18] [MUL 20] :

- Les événements sont des dispositifs de proximité géographique temporaires, virtuels ou non, permettant aux acteurs impliqués de communiquer, confronter leurs idées créatives [BAT 08]. Ils peuvent donc être vus comme des éléments importants pour le développement des écologies créatives [MUL 20], voire de régénération des territoires [PAI 08]. Ils peuvent prendre la forme de festivals [SAL 16], conventions et salons spécialisés [BAT 08] ou de concours [DEC 16].

- Les projets forment aussi une base de communication et de confrontation des idées créatives. À la différence des événements qui ne préjugent pas des caractéristiques des participants, les projets sont plutôt dirigés vers des groupes d'intérêts ou communautés et fournissent à leurs membres des opportunités pour aligner leurs visions et partager leurs expériences [GRA 13].

Un point important distinguant les différents niveaux économiques réside dans le niveau de proximité des acteurs avec les mécanismes marchands et redistributifs [COH 10]. De ce fait, nous pouvons nous attendre à constater une correspondance entre la dimension privilégiée de l'innovation, qu'elle soit principalement économique ou sociale, et le niveau économique concerné. Ainsi, en étant en contact direct avec les mécanismes de marché, les acteurs de l'upperground auront-ils tendance à insister sur la valeur économique, les amenant à privilégier la production d'innovations comportant une dimension économique forte. Ils seront particulièrement attentifs à leur capacité de valorisation suivant les mécanismes économiques traditionnels, qu'ils soient marchands (amélioration de la compétitivité, création de nouvelles niches, voire de nouveaux marchés...) ou redistributifs (meilleure prise en compte de besoins spécifiques par les administrés...). À l'inverse, les acteurs des niveaux économiques «inférieurs» (middleground et underground) sont plus éloignés des mécanismes marchands et redistributifs. Ils sont susceptibles de privilégier des logiques réciprocitaires ainsi que les dimensions extra-économiques des innovations : artistique, sociale, etc. De ce fait, il est attendu que ces acteurs développent des innovations privilégiant des enjeux et problèmes sociaux et pour lesquelles les enjeux de valorisation économique sont considérés comme secondaires. De ces attendus différents concernant les résultats découlent différents modèles de production et de gouvernance des innovations sociales qui seront abordés dans la section suivante.

\section{La production des innovations sociales : logiques «top-down » et « bottom-up »}

À l'instar de la plupart des innovations à dimension économique, les activités créatives sont consubstantielles de la production des innovations sociales et s'inscrivent dans des dynamiques collectives au sein d'écosystèmes impliquant des acteurs multiples. Peuvent ainsi être rencontrés 
différents types d'acteurs initiateurs. Hormis les entreprises commerciales et les institutions publiques et autres formes collectives plus informelles, les structures de l'économie sociale et solidaire (ESS, formées des coopératives, mutuelles, associations, fondations et entrepreneurs sociaux) sont souvent désignées comme étant à l'initiative des innovations sociales [RIC 09][PRA 15]. La capacité des structures de l'ESS à produire des innovations sociales est avant tout liée à leurs statuts, basés sur la libre adhésion, la gestion démocratique, la lucrativité limitée et la solidarité [RIC 12a]. Ceci implique qu'elles constituent des lieux d'expérimentations sociales où les processus de production des innovations n'ont pas pour finalité première la recherche d'un avantage d'ordre économique. Au contraire, leur activité d'innovation peut être sous-tendue par un objectif de prestation de services en faveur de leurs membres, ce qui est fréquemment le cas au sein des coopératives. Elles contribuent donc à l'intérêt commun. Dans d'autres cas, elles peuvent répondre à des besoins exprimés au sein de populations en situation de précarité, à l'instar des entrepreneurs sociaux. Leur fonction concourt donc à la recherche de l'intérêt général.

En outre, les dynamiques collectives à la base de l'innovation obéissent à différentes logiques que nous allons décrire dans cette partie. Nous pouvons identifier deux logiques créatives différentes, topdown et bottom-up. Chacune de ces deux logiques est déterminée par le type et la localisation des acteurs à leur initiative.

\subsection{La logique top-down}

Une première logique peut être qualifiée de top-down. L'initiative y est laissée aux structures de l'upperground. À ce titre, nous souhaitons insister sur deux types d'acteurs du secteur de l'ESS dont l'activité vise précisément à développer et mettre en œuvre des innovations sociales dans un contexte économique marchand.

- Un premier type est constitué des entrepreneurs sociaux. Ils désignent des individus ou des organisations mobilisant une logique commerciale en vue d'améliorer la situation de populations exclues, marginalisées ou souffrantes et qui n'ont pas les moyens d'améliorer leur situation [SAE 19].

- Un second type d'acteurs est constitué des structures de types fondations, relevant du mécénat de grandes structures privées (fondations d'entreprises...), publiques (fondations d'universités...) ou familiales. Il s'agit de structures à but non lucratif, ayant pour objectif la recherche de l'intérêt général. Elles se caractérisent par le fait que les mécènes sur lesquels elles s'adossent assurent leur viabilité économique, leur permettant ainsi de se consacrer entièrement à leur activité sociale. En contrepartie, elles sont tenues d'adopter des systèmes et pratiques de management les rapprochant de structures commerciales [RAE 18].

Malgré la diversité de leurs formes juridiques, les structures initiatrices des innovations sociales ont pour caractéristique commune d'être encastrées dans des relations économiques et institutionnelles complexes, comprenant différents types de structures publiques ou privées. Ces relations sont fondées sur des effets de complémentarité (par exemple, dans les cas de l'aide à l'insertion, la gestion et la valorisation du patrimoine culturel...) ou de substituabilité (par exemple, dans le secteur bancaire). De ce fait, elles bénéficient de ressources leur permettant de diffuser à grande échelle les innovations sociales produites. Un autre élément distinguant leur activité de celle des structures commerciales et publiques de l'upperground est de combiner création de valeur sociale et création de valeur économique [ACS 13]. Ces deux dimensions peuvent, suivant les cas, être sources de complémentarités mais aussi de tensions, lorsque les finalités associées sont divergentes [SAE 19]. En outre, leur capacité d'action peut être limitée par les attentes des financeurs [RAE 18][EYN 15]. Au final, il est attendu que les innovations sociales associées à une logique top-down aient la capacité de se diffuser à grande échelle au sein des systèmes socio-économiques, mais, en contrepartie, leur dimension sociale demeure limitée. En effet, en étant encastrées dans les systèmes socio-économiques 
existants, elles ne peuvent pas modifier de manière fondamentale les règles prévalant en leur sein, ce qui peut remettre en question l'apport réel de l'innovation pour le public ciblé [RIC 12b].

\subsection{La logique bottom-up}

Parallèlement à celles produites suivant une logique top-down, les innovations sociales se développent aussi suivant une logique bottom-up, aussi dit grass-root. Cette logique de production a été particulièrement étudiée en économie de la transition [SEY 12][SEY 13], ainsi que dans la littérature liant innovation sociale et développement territorial [NUS 04]. Les innovations sociales y sont produites par des réseaux d'activistes et organisations cherchant à trouver des solutions locales à des problèmes et besoins exprimés par les populations concernées. Elles passent donc par le développement et la mise en œuvre d'expérimentations à petite échelle [SEY 07]. De ce point de vue, ce type d'innovations sociales émane d'un underground constitué d'individus menant des activités au sein de structures, formelles ou non, n'ayant pas de visée marchande ou redistributive [COH 10]: collectifs de personnes, associations locales, etc. Au plus près de la population ciblée, cette logique privilégie une coconstruction avec cette dernière [YOU 19], tout en se fondant sur des mécanismes de réciprocité [HEN 05]. Son intérêt est que, en prenant pour point de départ la population ciblée, elle est censée être la plus à même de répondre à ses besoins.

En partant d'une échelle très restreinte (individuelle ou petit collectif), la limite de cette logique de production est que les innovations sociales peuvent avoir des difficultés à se diffuser et à faire l'objet d'une appropriation à des niveaux collectifs plus élevés. Les innovations sociales provenant de l'underground doivent dès lors faire l'objet au préalable d'un travail de traduction au sens de Callon et Latour [CAL 91] afin qu'elle puisse être comprise au niveau de l'upperground. En effet, pour pouvoir s'étendre et produire leurs effets, elles ont besoin d'un soutien actif de l'upperground se matérialisant, par exemple, par la fourniture de ressources financières, matérielles ou relationnelles [GRA 13]. De plus, les innovations sociales étant encastrées dans leur contexte de production, il devient nécessaire de mener un travail visant à étendre leurs effets à une échelle plus large. Ceci implique la mise en œuvre de stratégies particulières, pouvant se traduire par une décontextualisation, voire une standardisation des solutions initiales. Ceci peut être sources de tensions au sein des structures initiatrices, voire avec les bénéficiaires, car les modifications impliquées peuvent être considérées comme donnant lieu à leur dénaturation [MOO 15].

$\mathrm{Au}$ final, les logiques top-down et bottom-up de production des innovations sociales montrent chacune des forces et faiblesses qui sont mutuellement complémentaires. Ainsi, la logique top-down est en mesure de donner lieu à des innovations sociales aisément diffusables du fait de la proximité des organisations instigatrices avec les mécanismes marchands et redistributifs. Cependant, le fait qu'elles cherchent à s'adresser à des populations importantes conduit leurs instigateurs à proposer des solutions plus générales, voire «standardisées », ce qui peut donner lieu à des difficultés à répondre aux besoins spécifiques des populations concernées. À ce problème de standardisation vient se greffer l'enjeu de viabilité économique imposé par les financeurs des organisations instigatrices. Ceci risque donc de limiter l'apport social des innovations proposées. À l'inverse, la logique bottom-up part des besoins réels exprimés par les individus et populations bénéficiaires et vise à proposer des solutions locales, encastrées dans leur contexte de production. Néanmoins, cet encastrement contextuel, souvent aggravé par une faiblesse dans les ressources disponibles, a pour effet d'obérer leurs capacités de diffusion à plus grande échelle.

De ce fait, compte tenu de leurs forces et faiblesses respectives, il est fréquent que les logiques topdown et bottom-up soient mobilisées de manière simultanée et complémentaire dans les processus de production des innovations sociales. Ceci est, par exemple, constaté dans le contexte de la mobilité en France, avec, d'une part, le développement de plans et de plateformes étatiques visant à favoriser les initiatives publiques et privées et, d'autres, le développement de solutions locales de mobilité par les publics concernés [SZO 18]. Cette mobilisation simultanée soulève plusieurs problèmes, parmi 
lesquels : comment garantir leurs complémentarités, tant en termes de populations couvertes, que de besoins adressés ? Comment régler les problèmes éventuels de répartition des ressources, ceci afin de circonscrire au maximum les effets délétères d'une mise en concurrence entre ces logiques ? En bref, la mise en œuvre simultanée des différentes logiques d'innovation sociale au sein d'une même écologie créative soulève le problème de sa gouvernance. La prochaine section donnera des éléments de réponse en mettant en exergue le rôle tenu par les structures de l'économie sociale et solidaire. Ces dernières constituent en effet des acteurs centraux de l'innovation sociale, non seulement, comme nous l'avons $\mathrm{vu}$, en étant actrices de leur développement, mais aussi en jouant un rôle important dans leur gouvernance.

\section{Les rôles des structures de l'ESS comme intermédiaires des innovations sociales}

La section précédente a développé l'idée que la production des innovations sociales s'inscrit dans des écologies créatives au sein desquelles les structures de l'ESS sont pleinement impliquées en tant qu'actrices de leur développement. Cette section cherche, à son tour, à mieux cerner les facteurs soustendant leur contribution à la gouvernance des écologies créatives. Nous défendons l'idée qu'elles y parviennent au travers d'une activité d'intermédiation relevant du middleground. En effet, en étant des lieux d'expérimentation sociale, les structures de l'ESS ont la possibilité de faciliter la diffusion des innovations sociales provenant de l'underground, soit vers d'autres écologies créatives et populations potentiellement intéressées, soit vers l'upperground. De ce fait, elles ont la capacité de concilier les logiques top-down et bottom-up au sein des écologies créatives associés aux innovations sociales, participant ainsi à leur gouvernance.

De manière plus précise, nous considérons les structures de l'ESS comme des intermédiaires «sujets », s'apparentant aux intermédiaires d'innovation, définis par Howells [HOW 06, p. 720] comme «une organisation agissant comme agent ou courtier dans tout aspect du processus d'innovation entre deux ou plusieurs parties ${ }^{1} »$. Contrairement à d'autres dispositifs d'intermédiation «objets», telles les plateformes (places de marché, forums, réseaux sociaux, etc.), ce type d'intermédiation est compatible avec la logique bottom-up associée à l'innovation sociale dans la mesure où elle ne confère qu'un potentiel dont la réalisation incombe aux parties prenantes de cette dernière. Elles restent ainsi tout à fait en mesure de le mobiliser suivant leurs propres besoins et impératifs. Leur contribution recouvre différentes formes complémentaires, se situant aussi bien à un niveau relationnel que dans la circulation des ressources, informations et connaissances [MUL 19].

D'un point de vue relationnel, de par leur proximité et leur capacité à mobiliser des acteurs disparates (acteurs publics et privés, publics bénéficiaires, etc.) provenant des différents niveaux économiques (upper-, middle- et underground), les structures de l'ESS ont la capacité de faciliter l'adaptation des structures de réseaux sociaux [voir NOO 99]. Elles sont ainsi en mesure de sélectionner et de mettre en relation des acteurs socialement distants et dont les ressources et connaissances peuvent être complémentaires mais absentes localement. Cette fonction s'apparente à celle de tertius gaudens, décrite notamment par Padgett et Ansell [PAD 93]. Dans le cas des innovations sociales, cette fonction permet en particulier à des membres de groupes sociaux marginalisés d'accéder à des acteurs et ressources qui auraient été inatteignables autrement. Les structures de l'ESS ont aussi la faculté de jouer le rôle de tiers de confiance, leur permettant ainsi d'agir comme des tertius iungens [LER 14]. Ces derniers contribuent à densifier un réseau local en réduisant les risques de hold-up associés à la mobilisation d'investissements spécifiques [NOO 99]. Ils contribuent ainsi à fluidifier les coordinations au sein de l'écologie et, ce faisant, sa gouvernance [OBS 05].

\footnotetext{
${ }^{1}$ Citation : "an organization or body that acts an agent or broker in any aspect of the innovation process between two or more parties", traduction de l'auteur.
} 
Les structures de l'ESS peuvent aussi assurer leur rôle d'intermédiation de l'innovation sociale en facilitant la circulation des connaissances et des ressources [MOR 08] et, donc, la diffusion des innovations sociales. Elles y parviennent en étant des espaces cognitifs communs, voire en jouant le rôle de brokers [MUL 19]. Ces derniers sont entendus comme facilitant les transactions [MAR 82] ainsi que la circulation de ressources entre différents acteurs [GOU 89]. À ce titre, elles ont la capacité d'organiser les interactions entre les membres afin de favoriser leurs proximités, notamment cognitive et institutionnelle. Pour ce faire, elles sont à l'origine de projets collectifs mobilisant des acteurs relevant des différentes strates économiques [LER 14][MUL 19]. La réduction des distances cognitives, la mise en commun de ressources et la production de règles communes constituent alors des enjeux majeurs qui peuvent être portés par l'intermédiation des structures de l'ESS [MAI 13].

Au final, les structures de l'ESS facilitent la circulation de ressources diverses, qu'il s'agisse d'idées et connaissances ou de ressources financières et physiques. Dans le même temps, elles «traduisent», adaptent et organisent les innovations sociales de manière à faire l'objet d'une appropriation par les structures de l'upperground. Ceci permet, non seulement de faciliter leur financement, mais aussi leur diffusion à une plus grande échelle.

\section{Conclusion}

Bien qu'elle mobilise des processus de production similaires aux innovations à dimension économique, l'innovation sociale s'en démarque par le fait que, par définition, son objectif principal ne réside pas dans la recherche d'un avantage concurrentiel ou la création de nouveaux marchés. Au contraire, il réside dans la satisfaction de besoins sociaux non couverts par les mécanismes économiques traditionnels. Par la mobilisation du modèle de l'écologie créative, nous avons mis en avant que leur production donne lieu à des processus collectifs impliquant différents types d'acteurs (publics concernés, acteurs à leur instigation, structures de soutien et de diffusion des innovations), relevant de niveaux économiques distincts et caractérisés par des rationalités et logiques d'action différentes. Si les innovations émanant de l'upperground sont soumises à un arbitrage entre viabilité économique et impact social, celle-ci est considérée comme secondaire dans le cas où elles proviennent de l'underground. Néanmoins, les faiblesses et complémentarités respectives de ces deux logiques donnent souvent lieu à une mise en œuvre conjointe, ce qui soulève dès lors l'enjeu de la gouvernance des processus collectifs conduisant à leur production. Au-delà des problèmes classiques de gouvernance, cet enjeu est particulièrement sensible dans le cas de l'innovation sociale, du fait de ses particularités la distinguant des innovations à dimension économique. Ceci nous a amenés à mettre en avant le rôle que peuvent jouer les structures de l'économie sociale et solidaire pour y répondre. Tirant notamment parti de leur positionnement au sein du middleground, ces dernières sont capables de jouer le rôle d'intermédiaires facilitant la diffusion des innovations sociales de l'underground, aussi bien par le biais d'une intermédiation relationnelle, qu'en facilitant la circulation des idées créatives et des connaissances.

La position particulière occupée par les structures de l'économie sociale et solidaire, entre middleground et upperground, est aussi source d'enjeux importants, relatifs, non seulement, à leur organisation interne, mais aussi au développement du secteur. Nous souhaitons ainsi souligner l'enjeu particulier de la professionnalisation du secteur, vis-à-vis de la production des innovations sociales. Cette professionnalisation est rendue nécessaire, premièrement, par le tournant entrepreneurial pris par le secteur au cours des dernières années, institutionnalisé dans la Loi ESS française de $2014^{2}$. De la même manière, leur position, en contact avec l'upperground, notamment à des fins de financement, les conduit à se plier à l'exigence d'adopter les pratiques managériales issues du monde économique [EYN 15]. Néanmoins, cette professionnalisation du secteur ne doit pas être menée au détriment des

\footnotetext{
${ }^{2}$ L'alinéa 1 de l'article 1 de la Loi ESS de 2014 dispose en effet que « L'économie sociale et solidaire est un mode d'entreprendre et de développement économique adapté à tous les domaines de l'activité humaine... ».
} 
valeurs et finalités sociales [DUP 15]. Le risque encouru serait, dès lors, celui de la normalisation du secteur vis-à-vis des mécanismes de marché et, donc d'un amoindrissement de sa capacité à développer et à porter les innovations sociales.

\section{Bibliographie}

[ACS 13] ACS ZJ., BOARDMAN MC, MCNEELY CL. «The Social Value of Productive Entrepreneurship ». Small Business Economics, vol. 40, n³, p. 785-796. 2013

[AND 09] ANDRÉ I., BRITO HE., MALHEIROS J. « Inclusive places, arts and socially creative milieux ». In Social innovation and territorial development, dans D. MACCALLUM, F. MOULAERT, J. HILLIER, ET S. VICARI HADDOCK, Ashgate, Farnham, 2009.

[BAT 17] BATHELT H., COHENDET P., HENN S, SIMON L. The Elgar Companion to Innovation and Knowledge Creation. Edward Elgar Publishing, Cheltenham, Northampton, 2017.

[BAT 08] BATHELT, H., SCHULDT N. « Between Luminaires and Meat Grinders: International Trade Fairs as Temporary Clusters ». Regional Studies, vol. 42, n 6 , p. 853-868. 2008.

[BOU 06] BOUCHARD MJ. «L’innovation sociale en économie sociale ». R-2006-01. Collection Recherche. Chaire de recherche du Canada en économie sociale, Université de Montréal. 2006.

[BOU 09] BOURDEAU-LEPAGE L., HURIOT J-M. « Proximités et interactions : une reformulation ». Géographie, économie, société, vol. 11, n³, p. 233-249. 2009.

[BUR 13] BURGER-HELMCHEN T. The Economics of Creativity: Ideas, Firms and Markets. Routledge, Londres. 2013

[CAJ 14] CAJAIBA-SANTANA G. «Social Innovation: Moving the Field Forward. A Conceptual Framework ». Technological Forecasting and Social Change, vol.82, p. 42-51. 2014.

[CAL 91] CALLON M., LATOUR B. La science telle qu'elle se fait: anthologie de la sociologie des sciences de langue anglaise. La Découverte, Paris. 1991

[CHO 15] CHOI N., MAJUMDAR S. « Social Innovation: Towards a Conceptualisation », dans S. MAJUMDAR, S. GUHA, N. MARAKKATH, Technology and Innovation for Social Change, Springer New Delhi. 2015.

[COH 10] COHENDET P., GRANDADAM D., SIMON L. « The Anatomy of the Creative City ». Industry \& Innovation, vol. 17, $\mathrm{n}^{\circ} 1$, p. 91-111, 2010.

[COH 14] COHENDET P., GRANDADAM D., SIMON L., CAPDEVILA I. « Epistemic communities, localization and the dynamics of knowledge creation ». Journal of economic geography, vol. 14, n 5, p. 929-954, 2014.

[DEC 16] DECHAMP, G., SZOSTAK B. «Organisational Creativity and the Creative Territory: The Nature of Influence and Strategic Challenges for Organisations ».M@n@gement vol. 19, n², p. 61-88, 2016.

[DUP 15] DUPUIS JC, BAYLE E. Management des entreprises de l'économie sociale et solidaire : identités plurielles et spécificités. De Boeck, Bruxelles, 2015.

[EYN 15] EYNAUD P., MOUREY D. « Apports et limites de la production du chiffre dans l'entreprise sociale ». Revue française de gestion, $\mathrm{n}^{\circ} 247$, p. 85-100, 2015.

[FIS 11] FISHER CM., AMABILE TM. «Creativity, improvisation and organizations », dans T. RICKARDS, MA. RUNCO, S. MOGER. The Routledge Companion to Creativity, Routledge, London, New York, 2011.

[FLO 05] FLORIDA, R. The flight of the creative class: the new global competition for talent. HarperBusiness, New York, 2005.

[GOD 12] GODIN B. « Social Innovation: Utopias of Innovation from c.1830 to the Present ». Working Paper 11. Project on the Intellectual History of Innovation. 2012.

[GOU 89] GOULD R., FERNANDEZ R. « Structures of Mediation: A Formal Approach to Brokerage in Transaction Networks ». Sociological Methodology vol.19, n¹, p. 89-126, 1989.

[GRA 13] GRANDADAM D., COHENDET P., SIMON L. «Places, spaces and the dynamics of creativity: The video game industry in Montreal ». Regional studies, vol. 47, ${ }^{\circ} 10$, p. 1701-1714, 2013.

[HAR 06] HARRISSON D., VEZINA M. « L'innovation sociale: une introduction ». Annals of Public and Cooperative Economics vol. 77, n², p. 129-138, 2006. 
[HAZ 03] HAZEL KL., ONAGA E. « Experimental Social Innovation and Dissemination: The Promise and Its Delivery ». American Journal of Community Psychology, vol. 32, n³-4, p. 285-294, 2003.

[HEN 05] HENRICH J., BOYD R., BOWLES S., CAMERER C., FEHR R., GINTIS H., MCELREATH R. « "Economic Man" in Cross-Cultural Perspective: Behavioral Experiments in 15 Small-Scale Societies ». Behavioral and Brain Sciences vol. 28, nº ${ }^{\circ}$, p. 795-815. 2005.

[HIL 04] HILLIER J. MOULAERT F., NUSSBAUMER J. « Trois essais sur le rôle de l'innovation sociale dans le développement territorial, Abstract ». Géographie, économie, société vol. 6, n², p. 129-152. 2004.

[HOW 06] HOWELLS J. «Intermediation and the role of intermediaries in innovation ». Research Policy, vol. 35, n ${ }^{\circ}$, p. 715-28, 2006.

[LAN 18] LANGE B., SCHÜßLER E. « Unpacking the Middleground of Creative Cities: Spatiotemporal Dynamics in the Configuration of the Berlin Design Field ». Regional Studies, vol. 52, n¹1, p. 1548-1558. 2018.

[LAV 01] LAVILLE J-L. « Vers une économie sociale et solidaire ? RECMA - Revue Internationale De L'Économie Sociale, $\mathrm{n}^{\circ} 281$, p. 39-53, 2001.

[LEV 08] LE VELLY, R. « La détermination du prix équitable ». Gestion, vol. 33, n 1, 59-65, 2008.

[LER 14] LEROUX I., MULLER P., PLOTTU B., WIDEHEM C. « Innovation ouverte et évolution des business models dans les pôles de compétitivité : le rôle des intermédiaires dans la création variétale végétale ». Revue d'Economie Industrielle, $\mathrm{n}^{\circ} 146$, p. 115-142, 2014.

[LES 17] LESLIE D., RANTISI NM. «Innovation and Cultural Industries ». dans H. BATHELT, P. COHENDET, S. HENN ET L. SIMON, The Elgar Companion to Innovation and Knowledge Creation, Edward Elgar Publishing, Cheltenham, 2017.

[MAI 13] MAISONNASSE J., RICHEZ-BATTESTI N., PETRELLA F. « La petite fabrique de la médiation territorialisée : vers un modèle multi partie prenante ? » Revue Interventions économiques, $\mathrm{n}^{\circ} 48$, http://interventionseconomiques.revues.org/2055, 2013.

[MAR 82] MARSDEN PV. 1982. «Brokerage behavior in restricted exchange networks », dans PV. MARSDEN ET N. LIN, Social structure and network analysis, Sage, Beverly Hills, 1982.

[MEH 17] MEHOUACHI C., GRANDADAM D., COHENDET P., SIMON L. «Creative Capabilities and the Regenerative Power of Creative Industries: Local and Global Ingredients », dans M. WAGNER, J. VALLS-PASOLA ET T. BURGER-HELMCHEN, The Global Management of Creativity, Routledge, London, New York, 2017.

[MOO 15] MOORE ML., RIDDELL D., VOCISANO D. « Scaling Out, Scaling Up, Scaling Deep: Strategies of Nonprofits in Advancing Systemic Social Innovation ». The Journal of Corporate Citizenship, n 58, p. 67-84, 2015.

[MOR 08] MORRISON A. « "Gatekeepers of Knowledge” within Industrial Districts: Who They Are, How They Interact ». Regional Studies, vol. 42, n6, p. 817-835, 2008.

[MOU 09] MOULAERT F. « Social innovation: institutionally embedded, territorialy (re)produced». In Social innovation and territorial development, dans D. MACCALLUM, F. MOULAERT, J. HILLIER, ET S. VICARI HADDOCK, Ashgate, Farnham, 2009.

[MUL 20] MULLER P., SZOSTAK B., BURGER-HELMCHEN T. «Le rôle d'intermédiation des activités entrepreneuriales du middleground dans la circulation des idées créatives. Le cas du Krautrock ». Revue internationale P.M.E, à paraître, 2020.

[MUL 19] MULLER P., TANGUY C. «Les organisations de l'économie sociale et solidaire (ESS) comme intermédiaires de l'innovation sociale : leurs apports... et limites ». Innovations, n58, p. 189-217, 2019.

[MUM 02] MUMFORD MD. «Social Innovation: Ten Cases From Benjamin Franklin », Creativity Research Journal, vol. 14, n², p. 253-266, 2002.

[MUM 03] MUMFORD MD., MOERTL P. « Cases of Social Innovation: Lessons From Two Innovations in the 20th Century », Creativity Research Journal vol. 15, n²-3, p. 261-266, 2003.

[NOO 99] NOOTEBOOM B. «The triangle: the roles of the go-between ». dans S. GABBAY ET R. LEENDERS, Corporate Social Capital and Liability, Kluwer, Dordrecht, 1999.

[NUS 04] NUSSBAUMER J., MOULAERT F. «Integrated Area Development and Social Innovation in European Cities: A Cultural Focus ». City, vol. 8, n² 2, p. 249-257, 2004.

[OBS 05] OBSTFELD D. « Social Networks, the Tertius Iungens Orientation, and Involvement in Innovation ». Administrative Science Quarterly, vol. 50, n¹, p. 100-130, 2005. 
[PAD 93] PADGETT JF., ANSELL CK. «Robust Action and the Rise of the Medici, 1400-1434 ». American Journal of Sociology vol. 98, n6, p. 1259-1319, 1993.

[PAI 08] PAIOLA M. « Cultural Events as Potential Drivers of Urban Regeneration: An Empirical Illustration ». Industry \& Innovation, vol. 15, n 5, p. 513-529, 2008.

[POL 09] POL E., VILLE S. « Social Innovation: Buzz Word or Enduring Term? » The Journal of Socio-Economics, vol. $38, \mathrm{n}^{\circ} 6$, p. 878-885, 2009.

[PRA 15] PRADES J. « Du concept d'« innovation sociale » ». Revue internationale de l'économie sociale : Recma, $\mathrm{n}^{\circ} 338$, p. 103-112, 2015.

[RAE 18] RAEDERSDORF S. « La construction d'un outil de contrôle de gestion innovant dans l'économie sociale et solidaire : le cas de la fondation Apprentis d’Auteuil ». Innovations, n57, p. 109-36, 2018.

[RIC 09] RICHEZ-BATTESTI N., VALLADE D. «Économie sociale et solidaire et innovation sociale : Premières observations sur un incubateur dedié en Languedoc Roussillon ». Innovations, n³0, p. 41-69, 2009.

[RIC 12a] RICHEZ-BATTESTI N., PETRELLA F., VALLADE D. «L'innovation sociale, une notion aux usages pluriels : Quels enjeux et défis pour l'analyse ?»Innovations, n³8, p. 15-36, 2012.

[RIC 12b] RICHEZ-BATTESTI N., VALLADE D. «Éditorial. Innovation sociale, normalisation et régulation ». Innovations, $\mathrm{n}^{\circ} 38$, p. 5-13, 2012.

[SAE 19] SAEBI T., FOSS NJ., LINDER S. « Social Entrepreneurship Research: Past Achievements and Future Promises ». Journal of Management, vol. 45, $\mathrm{n}^{\circ} 1$, p. 70-95, 2019.

[SAL 16] SALAUN V., FULCONIS F., FABBE-COSTES N. «Quels mécanismes au cœur des organisations temporaires pulsatoires? » Revue francaise de gestion, $\mathrm{n}^{\circ} 259$, p. 83-99, 2016.

[SAR 17] SARAZIN B., COHENDET P., SIMON L, Les communautés d'innovation : de la liberté créatrice à l'innovation organisée, Regards sur la pratique, Éditions EMS, Caen, 2017.

[SEY 12] SEYFANG G., HAXELTINE A. « Growing Grassroots Innovations: Exploring the Role of Community-Based Initiatives in Governing Sustainable Energy Transitions ». Environment and Planning C: Government and Policy, vol. 30, n³, p. 381-400, 2012.

[SEY 13] SEYFANG G., PARK JJ., SMITH A. «A Thousand Flowers Blooming? An Examination of Community Energy in the UK ». Energy Policy, vol. 61, p. 977-989, 2013.

[SEY 07] SEYFANG G., SMITH A. 2007. « Grassroots innovations for sustainable development: Towards a new research and policy agenda ». Environmental Politics, vol. 16, n4, p. 584-603, 2007.

[SIM 09] SIMON L. « Underground, upperground et middle-ground: les collectifs créatifs et la capacité créative de la ville ». Management international, vol. 13, p. 37-51, 2009.

[STO 06] STOLARICK K., FLORIDA R. « Creativity, Connections and Innovation: A Study of Linkages in the Montréal Region ». Environment and Planning A, vol. 38, n¹0, p. 1799-1817, 2006.

[SZO 18] SZOSTAK B., MULLER P., FAGBOHOUN S., YAHIAOUI S. « Caractériser la gouvernance d'un écosystème d'innovation ouverte fondé sur des communs. Exploration du cas du transport et de la mobilité en France ». 9èmes Journées du GT Innovation de l'AIMS, Montréal, 17-19 octobre, 2018

[TOR 08] TORRE A. « On the Role Played by Temporary Geographical Proximity in Knowledge Transmission ». Regional Studies, vol. 42, nº, p. 869-889, 2008.

[VAN 16] VAN DER HAVE RP., RUBALCABA L. «Social Innovation Research: An Emerging Area of Innovation Studies? » Research Policy, vol. 45, n 9, p. 1923-1935, 2016.

[YOU 19] YOUNES D., JACOB M-R., MARTI I. « L'innovation sociale sur les territoires - Comment passer de l'intervention exogène à la communauté innovante ? » Revue Française de Gestion, n²80, p. 75-90, 2019.

[ZES 11] ZESCHKY M., WIDENMAYER B., GASSMANN O. «Frugal Innovation in Emerging Markets ». ResearchTechnology Management, vol. 54, nº, p. 38-45, 2011. 